\title{
RBF network based integral backstepping sliding mode control for USV
}

\author{
Renqiang Wang ${ }^{\mathrm{a}}$, Hua Deng, Keyin Miao, Yue Zhao, Jiabao Du \\ Navigation College, Jiangsu Maritime Institute, Nanjing 211170, PR China
}

\begin{abstract}
A kind of USV course RBF network control algorithm is putted forward, which is on the basis of integral backstepping sliding mode. First of all, an integrator sliding surface were designed with the sliding mode variable structure control technology. Secondly, radial basis function neural network was applied to approximate the system nonlinear function and uncertain parameters. Furthermore, a nonlinear damping law was introduced to overcome the bounded outside interference. Finally, on the basis of the above, the system control law was deduced by using the backstepping method. The simulation results show that the neural network can accurately approximate the nonlinear function and uncertain parameters, and the controller output is smooth and the output is not sensitive to perturbation of parameters. Therefore, the proposed algorithm is effective for USV course control.
\end{abstract}

\section{Introduction}

Maritime shipping and production activities are becoming more and more busy, the Unmanned Surface Vehicle (USV) due to the advantages of high nautical rate, high efficiency, low cost, severe sea condition that can instead people to the various activities has become an important development trend of the future of the ship. Therefore, USV is playing increasing roles in commercial, scientific and military applications [1]. Due to the disturbances of wind, wave, current and other marine environment, the USV will inevitably deviate from the original given course when it is sailing. Precise course control is a prerequisite to solve the trajectory tracking, autonomous navigation and collision avoidance and other issues. The design of ship course controller is the issues of common concern for the control theory and control engineering [2]

With the development of computer technology and modern control theory, a variety of new control algorithms, such as the Lyapunov's direct method [3], backstepping control [4-6], sliding mode control [7-9], fuzzy control [10-12] and neural network control [13-16] have been applied to the research of ship course control. It is well known that the most advantage of sliding mode control is able to overcome uncertainty of the system, which has highly robustness for uncertain dynamic system with outside disturbance, especially effectiveness for control of modelless system. It is an important method for suppressing high frequency switching that causes chattering of control input. And the variable structure control system algorithm is simple, fast response, and robustness to external noise and parameter perturbation.

\footnotetext{
a Corresponding author: wangrenqiang2009@126.com
}

Therefore, it is useful for ship steering control. The integral link joined into the algorithm of sliding mode control can also effectively eliminate the bounded interference outside so that the original controller has a stronger anti-jamming capability.

In this paper, by taking the "Lanxin" USV of Dalian maritime university as the research object, aiming at its motion control system, the nonlinear mathematical model for the USV planar motion is established by the method of responsive integrated backstepping modeling. Based on the Nomoto mathematical model and the combination of integral backstepping control, sliding mode control and neural network control, a kind of USV course RBF network control algorithm of USV course control is proposed. With the disturbances of wind, wave and current, simulation results show that the designed course controller can be properly adopted to the "Lanxin" USV course keeping with good effectiveness.

\section{Problem description}

In actual voyages, ship movements usually exhibit a nonlinear state. Therefore, in the mathematical model of USV plane motion, the nonlinear model is considered. Based on the linear mathematical model of plane three degrees of freedom, the nonlinear model is described by adding nonlinear term as following.

$$
\ddot{\varphi}+\frac{1}{T} H(\dot{\varphi})=\frac{K}{T} \delta+d(t)
$$

Where, $K$ and $T$ represent ship turning and follow index, 
$\varphi$ represents ship course, $\delta$ represents rudder angle, $d(t)$ represents ship external interference. And $H(\dot{\varphi})$ is nonlinear function with regard to $\dot{\varphi}$, $H(\dot{\varphi})=\alpha_{1} \dot{\varphi}+\alpha_{2} \dot{\varphi}^{3}+\alpha_{3} \dot{\varphi}^{5}+\cdots \quad$,where, $\alpha_{\mathrm{i}(\mathrm{i}=1,2,3, \cdots)}$ is the real value of the constant.

Make the following settings, $x_{1}=\varphi, x_{2}=\dot{\varphi}$, $u=\delta$. Then formula (1) can be converted into the following format:

$$
\left\{\begin{array}{l}
\dot{x}_{1}=x_{2} \\
\dot{x}_{2}=f\left(x_{2}\right)+g \cdot u+d(t) \\
y=x_{1}
\end{array}\right.
$$

Where, $f\left(x_{2}\right)=-\frac{1}{T} H(\dot{\varphi}), g=\frac{K}{T}, x_{1}$ and $x_{2}$ are system variable, $y$ is the actual output of the system, $f\left(x_{2}\right)$ is unknown function of system, $g$ is known gain of control input, $d(t)$ represents ship external interference, and $u$ is the control input of system.

Based on above, the goal of this article is to seek control law $u$ which is able to make the system output $y$ asymptotically tracking expected course $\varphi_{r}$, the tracking error will be infinitely close to zero, that is $e=y-\varphi_{r} \rightarrow 0$, and the stabilization time will be reduced as much as possible.

\section{Dteering control design based on backstepping}

The design steps of the ship course controller contain the following steps.

Step 1. According to the characteristics of system (2), the following sliding surfaces are defined.

$$
\left\{\begin{array}{l}
\dot{\xi}=z_{2} \\
z_{1}=x_{1}-\varphi_{r} \\
z_{2}=x_{2}-\sigma_{1}
\end{array}\right.
$$

Where, $\varphi_{r}$ is the expected course, $\sigma_{1}$ is virtual stabilization, $Z_{1}$ is the heading error. $\xi$ is the integral term which can be able to eliminate the static error caused by the uncertain interference term in the control process.

Step 2. Lyapunov function is constructed to prove the asymptotic stability control system by using backstepping method. The first Lyapunov functionis constructed.

$$
V_{1}=\frac{1}{2} z_{1}^{2}
$$

According to (3), it is obtained (5).

$$
\dot{z}_{1}=z_{2}+\sigma_{1}-\dot{\varphi}_{r}
$$

It can be inferred formula (6) by formula (4).

$$
\dot{V}_{1}=z_{1} \dot{z}_{1}=z_{1}\left[z_{2}+\sigma_{1}-\dot{\varphi}_{r}\right]
$$

The virtual stabilization $\sigma_{1}$ is set as formula (7)

$$
\sigma_{1}=-k_{1} z_{1}+\dot{\varphi}_{r}
$$

Therefore, it can be obtained (8).

$$
\dot{\sigma}_{1}=-k_{1}\left(-k_{1} z_{1}+z_{2}\right)+\ddot{\varphi}_{r}
$$

It can be obtained (9), according to (6) and (7).

$$
\dot{V}_{1}=-k_{1} z_{1}^{2}+z_{1} z_{2}
$$

Similarly, it is obtained (10) according to (3).

$$
\dot{z}_{2}=\dot{x}_{2}-\dot{\sigma}_{1}=f\left(x_{2}\right)+g \cdot u+d(\lambda)-\dot{\sigma}_{1}
$$

The second Lyapunov function is constructed.

$$
V_{2}=V_{1}+\frac{1}{2} z_{2}^{2}
$$

It can be inferred formula (12) by formula (11).

$$
\begin{aligned}
& \dot{V}_{2}=\dot{V}_{1}+z_{2} \dot{z}_{2}=-k_{1} z_{1}^{2}+z_{1} z_{2} \\
& +z_{2}\left(f\left(x_{2}\right)+g \cdot u+d(t)-\dot{\sigma}_{1}\right)
\end{aligned}
$$

And the third Lyapunov function is constructed.

$$
V_{3}=V_{2}+\frac{\lambda}{2} \xi^{2}
$$

It can be inferred formula (14) by formula (13).

$$
\begin{aligned}
& \dot{V}_{3}=\dot{V}_{2}+\lambda \xi \dot{\xi}=-k_{1} z_{1}^{2}+z_{1} z_{2}+\lambda \xi z_{2} \\
& +z_{2}\left(f\left(x_{2}\right)+g \cdot u+d(t)-\dot{\sigma}_{1}\right) \\
& =-k_{1} z_{1}^{2}+z_{2}\left(z_{1}+f\left(x_{2}\right)+g \cdot u+d(t)-\dot{\sigma}_{1}+\lambda \xi\right)
\end{aligned}
$$

Step 3. The RBF network is used to adapt the system function $f(x)$, and its algorithm is (15).

$$
\left\{\begin{array}{l}
h_{j}=\exp \left(-\frac{\left\|x-c_{j}\right\|^{2}}{2 b_{j}^{2}}\right), j=1,2, \cdots, m \\
f(x)=W^{*^{T}} \mathbf{h}(x)+\varepsilon
\end{array}\right.
$$

Where $\boldsymbol{x}$ is the network input, $i$ represents one of the first input network input layer, $j$ is a hidden layer of the network the first network input, $\boldsymbol{h}=\left[h_{j}\right]^{\mathrm{T}}$ is high 
Gaussian function, $\boldsymbol{W}^{*}$ is the ideal for weighting value, $\varepsilon$ is ideal for neural network approximation error.

$$
\hat{f}\left(x_{2}\right)=\hat{W}^{T} \mathbf{h}(\mathbf{x})
$$

Where, $\tilde{W}=\hat{W}-W^{*}$.

The global Lyapunov function is constructed.

$$
L=V_{3}+\frac{1}{2} \gamma \tilde{W}^{T} \tilde{W}
$$

It can be inferred formula (18) by formula (17).

$$
\dot{L}=\dot{V}_{3}+\gamma \tilde{W}^{T} \dot{\hat{W}}
$$

It can be obtained formula (19) by considering formula (14) and (16).

$$
\begin{aligned}
\dot{L}= & -k_{1} z_{1}^{2}+\gamma \tilde{W}^{T} \dot{\hat{W}} \\
& +z_{2}\left[\begin{array}{l}
z_{1}+f\left(x_{2}\right)+g \cdot u \\
+d(t)-\dot{\sigma}_{1}+\lambda \xi
\end{array}\right]
\end{aligned}
$$

Step 4. In order to eliminate the uncertain interference term in equation (19), the nonlinear damping law[9] was designed.

$$
u=\frac{1}{g}\left[-z_{1}-\hat{f}\left(x_{2}\right)-\left(k_{2}+\eta\right) z_{2}-\lambda \xi+\dot{\sigma}_{1}\right]
$$

It can be inferred formula (21) by considering formula (19) and (20).

$$
\begin{aligned}
\dot{L}= & -k_{1} z_{1}^{2}+\gamma \tilde{W}^{T} \dot{\hat{W}} \\
& +z_{2}\left[f\left(x_{2}\right) \hat{-f}\left(x_{2}\right)+d(t)-z_{2}\left(k_{2}+\eta\right)\right] \\
= & -k_{1} z_{1}^{2}-k_{2} z_{2}^{2}-\eta z_{2}^{2}+\tilde{W}^{T}\left(\gamma \dot{\hat{W}}-\mathbf{h} z_{2}\right) \\
& +[d(t)+\varepsilon] z_{2}
\end{aligned}
$$

In order to ensure system asymptotically stable, the following adaptive law is designed.

$$
\dot{\hat{W}}=\frac{1}{\gamma} \mathbf{h} z_{2}
$$

It is easy obtained formula (23) by theorem “ $x y \leq \eta x^{2}+\frac{1}{4 \eta} y^{2}$.

$$
\varpi z_{2} \leq \eta z_{2}^{2}+\frac{1}{4 \eta}\|\varpi\|_{\infty}^{2}
$$

Where, $\varpi=d(t)+\varepsilon$.

The fitness law of the network weights is still adopted formula (22), therefore, the formula (24) can be acquired.

$$
\begin{aligned}
\dot{L} & =-k_{1} z_{1}^{2}-k_{2} z_{2}^{2}-\eta z_{2}^{2}+\varpi z_{2} \\
& \leq-k_{1} z_{1}^{2}-k_{2} z_{2}^{2}+\frac{1}{4 \eta}\|\varpi\|_{\infty}^{2} \\
& \leq-k_{2} z_{2}^{2}+\frac{1}{4 \eta}\|\varpi\|_{\infty}^{2}
\end{aligned}
$$

In order to ensure that the system (2) is asymptotically stable, it is only need to prove the establishment which is $k_{2} z_{2}^{2} \geq \frac{1}{4 \eta}\|\omega\|_{\infty}^{2}$. Proof slightly, see the literature [4].

\section{Simulation}

USV course tracking control simulation is excuted by applying the above control algorithm.

\subsection{Simulation Object and Conditions Setting}

The establishment of USV planar motion mathematical model requires 8 known USV parameters, as shown in the table 1 .

Table 1. The "LanXin" USV Parameters

\begin{tabular}{c|c}
\hline Length Between Perpendiculars & $7.02 \mathrm{~m}$ \\
\hline Breadth & $2.60 \mathrm{~m}$ \\
\hline Speed & $<=35 \mathrm{kn}$ \\
\hline Draft (full load) & $0.32 \mathrm{~m}$ \\
\hline Block Coefficient & 0.6976 \\
\hline Displacement (full load) & $2.73 \mathrm{~m}^{3}$ \\
\hline Rudder Area & $0.2091 \mathrm{~m}^{2}$ \\
\hline $\begin{array}{c}\text { Distance Between Barycenter } \\
\text { and Center }\end{array}$ & $0.35 \mathrm{~m}$ \\
\hline
\end{tabular}

In this paper, the planer motion mathematical model for the "Lanxin" USV adopts Nomoto model from the nonlinear mathematical model of formula (1).

There is the formula of matrix parameters of the mathematical model of the ship to calculate the above parameters, When the speed is $8.5 \mathrm{kn}$, the gain constant and time constant are obtained through MATLAB programming, an $K=1.50226, T=0.90503, \alpha_{1}=0.001$, $\alpha_{i(i=2,3, \ldots)}=0$. They are the Nomoto model parameters of the "Lanxin" USV [17]. In the actual simulation, the influence of wind and wave for USV motion is obtained with white noise and a second-order wave' $s$ transfer function $[18,19]$. The formula can be written as

$$
y(s)=h(s) w(s)
$$

Where $w(s)$ is the zero-mean Gaussian white noise, the power spectral density is $0.1, h(s)$ is a second-order wave transfer function, and $h(s)$ can be written as formula (26). 


$$
\left\{\begin{array}{l}
h(s)=\frac{K_{\omega} s}{s^{2}+2 \xi \omega_{0} s+\omega_{0}{ }^{2}} \\
K_{\omega}=2 \xi \omega_{0} \sigma_{m}
\end{array}\right.
$$

Where $\omega_{0}$ is the dominant frequency of sea waves, $\xi$ is the damping coefficient, $K_{\omega}$ is the gain constant. $\sigma_{m}$ is the constant describing wave intensity.

Based on USV motion model, the controller adds the model of the rudder servo system as formula (27).

$$
\dot{\delta}=-\frac{1}{T_{r}} \delta+\frac{1}{T_{r}} \delta_{r}
$$

Where $T_{r}$ is time constant, for USV is generally about $0.2 \mathrm{~s}$. $\delta_{r}$ is Command rudder angle. There is the actually limit of rudder angle that is the formula (28).

$$
|\delta| \leq 35^{\circ}
$$

\subsection{Simulation Results and Analysis}

\subsubsection{Course changes experiment}

\section{(1) experiment of $20^{\circ}$ change of course}

Protocol initial heading as $000^{\circ}$, expected course $020^{\circ}$, the simulation results without external interference and model parameter perturb are shown in Fig.1.

It can be seen from Figure 1, the control effect is good,and the output of course will reach the expected value faster, costing 30s without overshoot nearly; and rudder control horn is reasonable which can meet the characteristics requirements of steering system.

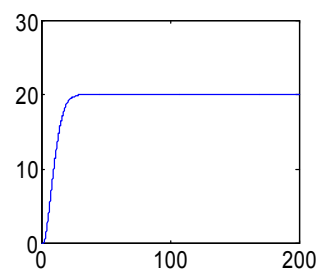

(a) course angle

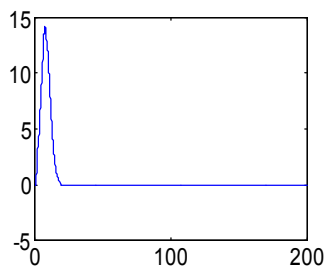

(b) rudder angle

\section{Fig.1. the output of course and rudder angle}

\section{(2) experiment of continuous change of course}

Conduct experiment of tracking square wave, set the cycle as $100 \mathrm{~s}$, the change of course as $030^{\circ}$, the experimental results will be shown in Fig.2.

It can be seen from Figure 2, in the case of desired track changes, the new controller can do better in steering and easing the helm counter steering and output the right rudder angle according to ship characteristics and sea condition at the right time, and also conduct without overshoot in Fig 2.

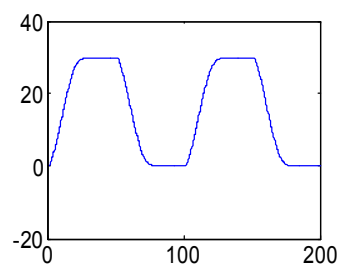

(a) course angle

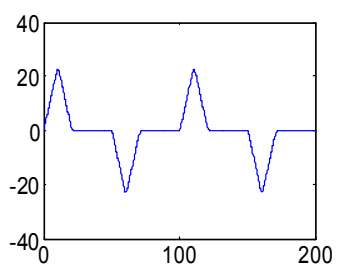

(b) rudder angle
Fig.2. the output of course and rudder angle when expected course continuous change

\subsubsection{Interference experiment}

\section{(1) experiments of white noise interference}

Protocol initial heading as $000^{\circ}$, desired track as $030^{\circ}$, apply amplitude as 0.1 white noiseinterference to course angle, the experiment results are shown in Fig.3.

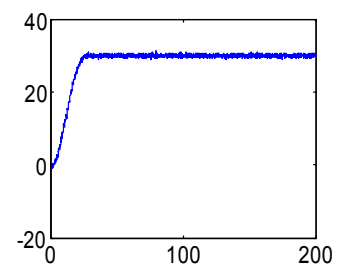

(a) course angle

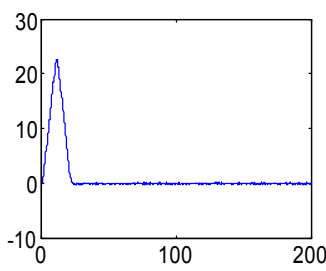

(b) rudder angle
Fig.3. the output of course and rudder angle under white noise interference

It can be seen from Figure 3, when the ship was disturbed by the outside world, the designed course controller will overcome outside interference to a certain degree, and will track the expected course with no shock of rudder controlling.

(2) experiments of ship parameter perturbation and white noise interference

Setting the protocol initial heading as $000^{\circ}$, desired track as $030^{\circ}$, propose maneuvering ship indices $\mathrm{K} 、 \mathrm{~T}$ perturb at $40 \%$, apply amplitude as 0.1 and make white noise interference to the course, the experiment results are shown in Fig 4.

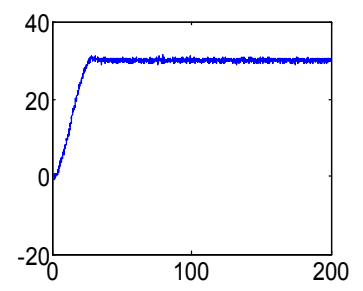

(a) course angle

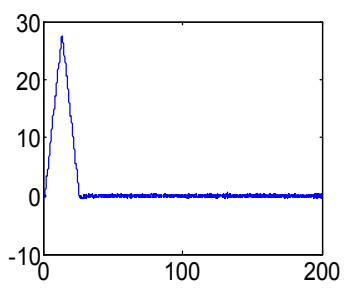

(b) rudder angle
Fig.4. the output of course and rudder angle under model parameter perturb and white noise interference 
It can be seen from Figure 4, when the ship model parameters occur perturbat with interference from the outside world, the course output from the new controller has smaller fluctuation around expect course, and has smaller shock amplitude of rudder controlling, thereby, it is illustrated that the anti-interference ability of the new controller is stronger.

\section{Conclusion}

An integral sliding mode controller with neural network was investigated for USV navigation, which realizes the accurate and stable tracking of the ship in the steering process, on the basis of backstepping method. With disturbances of wind, wave and current, the RBF network based integral backstepping sliding mode control system is simulated and the results of simulation show that the proposed algorithm is effective for USV course control.

\section{Acknowledgment}

This work was supported by the Foundation of Jiangsu Maritime Institute under Grant No.XR1501 and No.XR1504, and Jiangsu University Brand Professional Construction Project under Grant No. PPZY2015B177.

\section{References}

1. B. Volker, Unmanned Surface Vehicles - A Survey, Skibsteknisk Selskab, Copenhagen, Denmark, 2008.

2. S. Bennett. Nicolas Minorsky and the automatic steering of ships, IEEE Control Systems Magazine, 1984, 4(4) : 10-15.

3. S. Kumarawadu, K. Kumara, On the speed control for automated surface vessel operation, in Proceedings of the 2007 third international conference on information and automation for sustainability, Melbourne, Australia, 2007: 135-140.

4. Benaskeur A.R, Desbines A. Backstepping based adaptive PID control[C]. Proceedings of Control Theory and Applications. 2002, 149 (1): 54-59.

5. Y. Pan, D. Huang, Z. Sun, Backstepping adaptive fuzzy control for track-keeping of underactuated surface vessels, Control Theory and Applications, 2011, 7: 907-914.

6. Renqiang Wang, Yuelin Zhao, Keyin Miao. Application of Neural Network Minimum Parameter Learning Algorithm in Ship's Heading Tracking Control[C]. Proceedings of 9th International Symposium on Computational Intelligence and Design, 2016, 12: 135-139.

7. Wang Renqiang, Gong Jianyun, Zhao Yuelin.Neural Network Sliding Mode Control under New Reaching Law and Application[C], Proceedings of Advanced Information Technology, Electronic and Automation Control Conference, 2015, 12 : 911-917.

8. X. Bao, K. Nonami, Z. Yu, Combined yaw and roll control of an autonomous boat, in Proceedings of the
2009 IEEE International Conference on Robotics and Automation, Kobe, Japan, 2009: 188-193.

9. Wang Ning, Lv Shuailin, Liu Zhongzhong. Global finite-time heading control of surface vehicles[J], Neurocomputing, 2016, 175 (106) : 662-666.

10. K. Do, Z. Jiang, J. Pan, Underactuated ship global tracking under relaxed conditions, IEEE Trans. on Automatic Control, 47(9): 1592-1536, 2002.

11. L. Yuan, H. Wu, Multiple slidfing mode adaptive fuzzy controller for nonlinear marine autopilot systems, CAAI Transactions on Intelligent Systems, 2010, 4: 308-312.

12. T. Li, Y. Yang, B. Hong, et al, Robust adaptive fuzzy design for ships track-keeping control, Control Theory and Applications, 2007, 3: 445-448.

13. Guanshan $\mathrm{Hu}$, Yingbing Zhou. Application of Fuzzy Neural Network in Ship Course Control[J], Applied Mechanics and Materials, 2012, 1511(135) : 309-315.

14. Xin Wang, Tieshan Li, Weilin Luo. Direct Adaptive Neural Network Control for a Class of Ship Course Uncertain Discrete-time Nonlinear Systems[J], Marine Engineering Frontiers, 2013, 1(3) : 42-48.

15. Yang Wang, Chen Guo, Fuchun Sun. Dynamic neural fuzzified adaptive control of ship course with parametric modelling uncertainties[J], Int. J. of Modelling, Identification and Control, 2015, 13(4) : 251-258.

16. Wang Renqiang, Zhao Yuelin, Sun Jianming.Application of Optimized RBF Neural Network in Ship's Autopilot Design[C], Proceedings of IEEE Advanced Information Management, Communicates, Electronic and Automation Control Conference, 2016, 10: 1642-1646.

17. FAN Yunsheng, SUN Xiaojie, WANG Guofeng, GUO Chen. On Fuzzy Self-adaptive PID Control for USV Course[C], Proceedings of the 34th Chinese Control Conference, 2015, 7: 8472-8478.

18. T. Fossen, Guidance and control of ocean vehicles. England: John Wiley \& Sons LTD., 1994.

19. C. Yang, X. Jia, Y. Bi, Rudder Stabilization of Ships and its Robust Control. Dalian: Dalian Maritime University Press, 2011. 